\title{
Review of: "Global identification and mapping of socio-ecological production landscapes with the Satoyama Index"
}

\author{
Melissa Vogt
}

Potential competing interests: The author(s) declared that no potential competing interests exist.

Global application of the satoyama index, a locally developed index, to identify SEPL.

Thank you for the opportunity to provide comments for this research article.

\section{Comments focus on points for improvement.}

Crop diversity is important for cultural purposes but also for biodiversity.

The studies chosen to demonstrate one aspect of compositional diversity as less valuable for biodiversity do not represent studies that actually prove crop diversity as valuable for biodiversity. The point might also coincide with the behaviour of the index identified as problematic, where forest converted to grassland is considered to have a high value. The cause might actually be because of the importance given to landscape configuration heterogeneity (high habitat diversity) instead of composition. Both are important for biodiversity if not essential, and for the social aspects of the index. Definitions of composition used might have or might influence how compositional diversity is considered valuable to biodiversity.

Recognises the limitation of satellite data and information, and the lack of sensitivity to locally focused interests, particularly related to the social aspects of interest for SEPL or other such concepts of assessment approaches. The suggestion to use different resolutions and extent of hectares is rational. In field research might also have been suggested as complementary.

Similarly, recognises that histories of land use is important. One reason for their importance is to contextualise index values.

The suggestion to include in OECMs is agreed as a good entry point for mobilising interest and improving understanding. The socio aspects of SEPL do however require in field understandings that satellite images can not provide, even with resolutions and extents of hectares, and that international or local statistical or other demographic and population level information, used for global applicability of the index but requiring locally specific information, mightn't either. 
The above suggested points for improvement would need to be adequately applied and integrated or in the process of being integrated before such inclusion.

\section{Other comments}

'This means that SEPL are not recognised as important in a conventional conservation sense.' Agree, even when they are found in conventional conservation land use types.

Applicability for Australia or lack there of, is understood, however regions of configurational heterogeneity exist. Improved detail allowed by the index and satellite technology used might allow such assessments and valuing in the future. 